\title{
Enfermedad periodontal y COVID-19: factores de riesgo y mecanismos compartidos
}

\author{
Periodontal disease and COVID-19: risk factors and shared mechanisms
}

Tonatiuh Abimael Baltazar-Díaz, ${ }^{*}$ Ana Lourdes Zamora-Pérez ${ }^{\ddagger}$

\begin{abstract}
RESUMEN
La enfermedad periodontal comprende diferentes etapas dentro de su espectro, siendo todas ellas consecuencia de afecciones inflamatorias del periodonto, producto de un desbalance patológico de la microbiota oral. La pandemia actual de COVID-19, causada por SARS-CoV-2 tiene fuertes implicaciones en diversas áreas de la medicina; sin embargo, la entrada del virus a través de las vías respiratorias altas y la relevancia de la cavidad oral para el control de infecciones enfatizan la importancia de revisar los diferentes hallazgos en torno a COVID-19 y la salud bucodental. La presente revisión examina múltiples factores de riesgo comunes a la enfermedad periodontal y al desarrollo de COVID-19, en la que se inspeccionan diferentes hipótesis y mecanismos que podrían establecer asociaciones entre ambas patologías. La identificación de grupos de riesgo durante la pandemia es muy importante para establecer recomendaciones de salud pública, por lo que el conocimiento en torno a patologías de alta prevalencia, como la enfermedad periodontal, resulta de especial significancia.
\end{abstract}

Palabras clave: COVID-19, enfermedad periodontal, factores de riesgo, disbiosis.

\section{INTRODUCCIÓN}

A finales de 2019, los primeros casos de neumonía por SARS-CoV-2 fueron reportados. ${ }^{1}$ La enfermedad que este virus causa se denominó COVID-19. Al 12 de diciembre de 2020, se reportan más de 69 millones de casos en el

\section{ABSTRACT}

Periodontal disease comprises different stages, all of them being the consequence of inflammatory conditions of the periodontium, induced by a pathological imbalance of the oral microbiota. The current COVID-19 pandemic, caused by SARS-CoV-2 has strong implications in various areas of medicine, however, the entry of the virus through the upper respiratory tract and the relevance of the oral cavity for the control of infections emphasize the importance of reviewing the different findings around COVID-19 and oral health. This short review multiple risk factors common to periodontal disease and the development of COVID-19, in which different hypotheses and mechanisms that could establish associations between both pathologies are inspected. The identification of risk groups during the pandemic is very important to establish public health recommendations, that is why then knowledge about highly prevalent pathologies such as periodontal disease is of special significance.

Keywords: COVID-19, periodontal disease, risk factors, dysbiosis.

mundo, ${ }^{2}$ mientras que en México se reportan alrededor de 1,392,000 casos, con más de 120,000 fallecimientos. ${ }^{3}$ La enfermedad periodontal (EP) es un padecimiento muy común, que comienza con la inflamación de la gingiva iniciada por la disbiosis de la microbiota residente en el diente y la gingiva. Si esta inflamación no es tratada,

* Estudiante del Doctorado en Ciencias en Biología Molecular en Medicina, Instituto de Enfermedades Crónico-Degenerativas.

‡ Instituto de Investigación en Odontología, Departamento de Clínicas Odontológicas Integrales.

Centro Universitario de Ciencias de la Salud, Universidad de Guadalajara.

Recibido: 10 de marzo de 2021. Aceptado: 12 de mayo de 2021.

Citar como: Baltazar-Díaz TA, Zamora-Pérez AL. Enfermedad periodontal y COVID-19: factores de riesgo y mecanismos compartidos. Rev Mex Periodontol. 2021; 12 (1-3): 12-17. https://dx.doi.org/10.35366/102954 
progresa hacia periodontitis crónica, caracterizada por la pérdida de la gingiva, hueso y ligamento, creando las bolsas periodontales que pueden llevar a la pérdida de la pieza dental. ${ }^{4}$ Si bien, la EP puede clasificarse como crónica o agresiva, no existen biomarcadores que diferencien una etapa de la otra, por lo que se trata de un mismo espectro de la enfermedad. ${ }^{5}$ Diferentes estudios estiman su prevalencia desde un $44 \%$ hasta un $93 \%$, dependiendo de la población estudiada y definiciones operacionales empleadas. ${ }^{6-10}$ Según el Sistema de Vigilancia Epidemiológica de Patologías Bucales SIVEPAB 2019, de una población adulta de $143,995,41.7 \%$ tiene un periodonto sano, indicando además que la prevalencia de gingivitis es mayor a $50 \%$ en todos los grupos de edad. ${ }^{11}$

SARS-CoV- 2 es un $\beta$-coronavirus de RNA de cadena sencilla, séptimo miembro patógeno de la familia Coronaviridae, a la cual también pertenecen el SARS-CoV y MERS-CoV, causantes de emergencias mundiales en años anteriores. ${ }^{12}$ La entrada a las células de SARS-CoV-2 depende de la proteína $S$ viral, que se une a la enzima convertidora de angiotensina 2 (ACE2, por sus siglas en inglés) localizada en la membrana celular, que posteriormente es escindida por la proteasa transmembranal TMPRSS2 y por la furina, que permiten el ingreso del virus. ${ }^{13,14}$ Casi al comienzo de la pandemia, se determinaron grupos de riesgo para el desarrollo de COVID-19 severa, como pacientes con diabetes (DM), obesidad, enfermedad pulmonar obstructiva crónica (EPOC), edad avanzada, hipertensión, enfermedad renal crónica (ERC), tabaquismo y recientemente, se propone que los pacientes con EP podrían conformar otro grupo de riesgo. ${ }^{15-18}$

Algunas hipótesis sugieren que los pacientes con enfermedad periodontal (EP) pueden constituir un grupo de riesgo para el desarrollo de COVID-19 severa, por factores de riesgo comunes a otras comorbilidades presentes, pero también en cuanto a la sobreexpresión de citocinas proinflamatorias con la que cursa la EP. ${ }^{17-19}$ Se sabe que la EP se encuentra comúnmente asociada con factores de riesgo importantes para el desarrollo de COVID-19 severa, como DM u obesidad. ${ }^{16,20-23}$ Además, también se han encontrado asociaciones de la EP con otras comorbilidades ante COVID-19 como ERC, ${ }^{24-26}$ EPOC o tabaquismo. ${ }^{27-32}$

\section{Diabetes}

La diabetes mellitus tipo 2 (DM en lo subsecuente) describe un grupo de desórdenes metabólicos caracterizados por hiperglicemia, si no es tratada. ${ }^{33}$ En México, la DM es la segunda causa de muerte, con una prevalencia total de $13.7 \%{ }^{34}$ El papel de la DM como factor de riesgo ante la enfermedad periodontal ha quedado patente a través de numerosos estudios epidemiológicos y clínicos. ${ }^{20}$ Los mecanismos patogénicos propuestos que median entre la DM y la enfermedad periodontal incluyen una respuesta inmune celular deteriorada, daño tisular por hiperglicemia y disbiosis. ${ }^{35}$

Existe evidencia acumulada en cuanto a la severidad de la COVID-19 y su asociación con DM alrededor del mundo y en Latinoamérica. ${ }^{16,36-38}$ Diversos autores han reportado el aumento en la expresión de ACE2 en pacientes diabéticos y con otras enfermedades crónicas, así como su influencia en las complicaciones cardiovasculares relacionadas. ${ }^{39,40}$ Los niveles plasmáticos de furina, proteína involucrada en el ingreso del virus a la célula, se encuentran elevados en pacientes con DM. ${ }^{41}$ De manera interesante, Oliveira y colaboradore ${ }^{42}$ muestran un vínculo entre el sistema renina-angiotensina, la exacerbación de la respuesta inflamatoria y la pérdida ósea en modelos de diabetes inducida, mostrando además un aumento en la expresión de ACE2 en tejido gingival luego de la inducción de enfermedad periodontal. Debido a la alta prevalencia de DM en la población, y que constituye un factor de riesgo importante para el desarrollo de COVID-19 severa, su conjunción con EP representa un importante grupo en el que sería útil identificar los factores de riesgo.

\section{Obesidad}

La obesidad es un factor predictivo para el desarrollo de enfermedades crónicas, como enfermedad cardiovascular, DM, entre otras. ${ }^{43}$ La asociación entre EP y obesidad es positiva, aunque el papel causal es todavía discutido. En este sentido, se reporta un incremento de casi un tercio en la razón de probabilidades en la prevalencia de obesidad en sujetos con EP. ${ }^{21,22} \mathrm{Al}$ igual que en DM, el papel de la inflamación es muy importante. En la obesidad, los adipocitos secretan citocinas proinflamatorias como TNF- $\alpha$ e IL-6, que además de hallarse en niveles elevados en pacientes obesos, estimulan la producción de proteínas de fase aguda y causan alteraciones en la respuesta inmune, que a la postre incrementan la susceptibilidad a infecciones. ${ }^{23,44}$ Los patógenos inductores de la periodontitis contribuyen asimismo a la secreción de TNF- $\alpha$, que favorece a la destrucción ósea y la degradación del tejido conectivo. ${ }^{21}$

La obesidad es un factor de riesgo reconocido para el desarrollo de COVID-19 severa, además de ser un problema de salud pública particularmente en población mexicana. ${ }^{15,45}$ De manera similar a la DM, la obesidad también cursa con inflamación de bajo grado, donde existe una expresión incrementada de citocinas proinflamatorias que además de afectar la respuesta inmune, se 
ha observado que incrementan el riesgo de enfermedades pulmonares infecciosas como la influenza. ${ }^{46}$ De manera interesante, los niveles de expresión de ACE2 son más altos en tejido adiposo ${ }^{39}$ que en los pulmones, y estos niveles son comparables entre individuos obesos y no obesos; sin embargo, los pacientes obesos tienen un número mayor de adipocitos, con lo que incrementan el número de células que expresan ACE2. ${ }^{47}$ Durante el curso de la $E P$, la secreción de citocinas proinflamatorias asociada a la disbiosis puede sumarse a la existente por parte de los adipocitos en pacientes obesos, por lo que la asociación con COVID-19 puede representar un escenario de alto riesgo para un agravamiento de la enfermedad.

\section{Enfermedad renal crónica}

Existen estudios que señalan la asociación y las afectaciones que provoca la enfermedad renal crónica (ERC) en dientes, mucosa bucal, periodonto, glándulas salivales y la lengua, lo que tiene un efecto negativo en la salud bucal del paciente. ${ }^{24,25}$ En casos sujetos a hemodiálisis, se reporta una alta prevalencia de EP, así como de su severidad. ${ }^{24,26}$ Pocos estudios abordan los posibles mecanismos entre los factores patogénicos de la EP y la ERC, dado que la ERC incluye una multitud de factores de confusión. Sin embargo, un estudio señala la correlación entre una menor diversidad en la microbiota oral y los años de tratamiento en hemodiálisis. ${ }^{48}$

La ERC se ha señalado como una comorbilidad de riesgo ante COVID-19; ${ }^{16}$ sin embargo, existe evidencia discordante al respecto. El metaanálisis por Wang y colaboradores ${ }^{49}$ sugiere que no existe correlación entre la ECD y el desarrollo de COVID-19 severa. Por otro lado, otro metaanálisis ${ }^{50}$ en donde los datos se analizaron de manera individual, muestra una asociación significativa de la ERC con la severidad de COVID-19.

\section{EPOC y tabaquismo}

La EPOC se caracteriza por obstrucción persistente al flujo aéreo, por lo general progresiva y parcialmente reversible. Esta limitación está asociada a una reacción inflamatoria pulmonar exagerada frente al humo del tabaco y biomasa principalmente. ${ }^{51}$ Se ha señalado una asociación

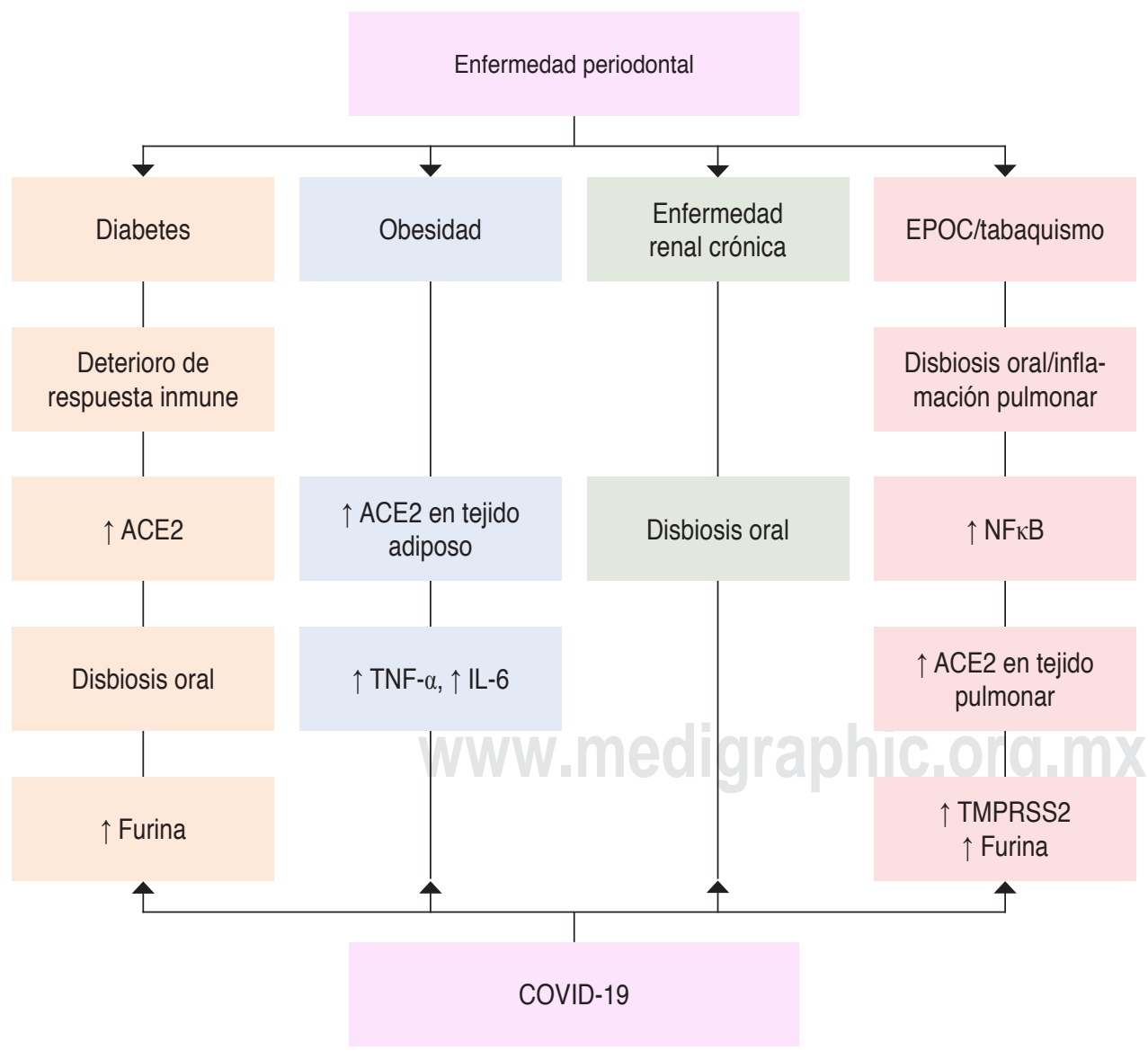

Figura 1:

Factores de riesgo comunes a enfermedad periodontal y COVID-19. En los cuadros, se señalan los mecanismos propuestos y eventos patológicos descritos por diferentes autores que podrían establecer asociaciones entre ambas patologías, no necesariamente causales. EPOC = enfermedad pulmonar obstructiva crónica, $\mathrm{ACE} 2$ = enzima convertidora de angiotensina 2, $\mathrm{NF} \times \mathrm{B}=$ factor nuclear kappa $\mathrm{B}$, TNF- $\alpha=$ factor de necrosis tumoral alfa, IL-6 = interleucina 6, TMPRSS2 $=$ proteasa transmembrana serina 2 . 
entre la EPOC y la EP, así como una correlación positiva en la severidad de ambas. ${ }^{52,53}$ Existe discusión respecto a si la EP constituye una comorbilidad ante la EPOC, o si es una mera asociación dados los factores de riesgo. Para explicar esto, se han señalado diversos mecanismos potenciales que conectan ambas patologías. El primero de ellos señala la aspiración de secreciones orales con bacterias potencialmente patógenas potenciadas por la disfunción inmune en el paciente con EP crónica. ${ }^{27,28}$ Otro mecanismo está mediado por la inflamación neutrofílica, la cual tiene un papel importante en el desarrollo y la progresión de la EPOC, ${ }^{54}$ correlacionada de manera íntima con el tabaquismo. Si bien la etiología primaria de la EP es la disbiosis oral, la respuesta inmune del hospedero también es fundamental en el establecimiento de la patología. En este sentido, varias revisiones destacan el papel de la respuesta inapropiada de los neutrófilos. ${ }^{29-31}$ Por ejemplo, la quimiotaxis de neutrófilos se ve afectada en pacientes con periodontitis crónica. ${ }^{32} \mathrm{Al}$ igual que los pacientes con EP, durante la EPOC los pacientes muestran mayores niveles de citocinas proinflamatorias como TNF- $\alpha$ y proteína C reactiva. ${ }^{31}$ Asimismo, se ha señalado que los neutrófilos provenientes de pacientes con EPOC tienen un mayor potencial proteolítico y quimiotáctico, que puede resultar perjudicial. ${ }^{31,55}$

La EPOC y el tabaquismo han sido señalados como factores importantes para el desarrollo de COVID-19 severa. ${ }^{56,57}$ Asimismo, se ha verificado que los pacientes con EPOC y con tabaquismo activo sobreexpresan ACE2 en las vías aéreas superiores, por lo que se propone que esto pudiera ser un mecanismo que explique el mayor riesgo de COVID-19 severa en estos pacientes. ${ }^{58}$ Por otro lado, esta elevación en la expresión de ACE2 en tejido pulmonar también fue verificada en personas con tabaquismo activo, pacientes con EPOC vs no fumadores, donde además de la elevación de ACE2, también se encontró un aumento de TMPRSS2 y furina. ${ }^{59}$ Por ello, la identificación de casos con factores de riesgo para el desarrollo de EPOC es importante, sumado al hecho de que son pacientes con un riesgo importante de presentar COVID-19 severa.

\section{Disbiosis}

La disbiosis oral es la interacción desequilibrada entre la microbiota oral y el hospedero, en detrimento del hospedero, y se ha señalado como la etiología principal en la EP. ${ }^{60-62}$ Tradicionalmente, se han identificado bacterias del denominado complejo rojo como responsables de la EP (Porphyromonas gingivalis, Tannarella forsythia y Treponema denticola), aunque esta lista se ha extendido a otras bacterias Gram negativas del filo
Firmicutes. ${ }^{62}$ Métodos de secuenciación masiva han ido apoyando la hipótesis de que la EP está dada por interacciones entre diferentes bacterias que después ocasionan alteraciones fisiológicas, en un modelo de sinergia polimicrobiana. ${ }^{61,63}$

El modelo de la sinergia polimicrobiana propone que la patogenicidad se inicia con microorganismos clave, que elevan la virulencia de la comunidad completa. Estos microorganismos se asocian con patógenos accesorios preexistentes, que facilitan la transición de la comunidad hacia una disbiosis que estimula la respuesta inflamatoria por parte del hospedero. En algunos individuos, esta respuesta puede estar mal regulada o mal dirigida, lo que contribuye a la destrucción del tejido, y retroalimenta patógenos con nutrientes derivados del tejido degradado (colágeno, compuestos derivados del grupo hemo, etcétera), formando una comunidad bacteriana que exacerba el proceso de la enfermedad ${ }^{61,62,64}$ y que además puede evadir la respuesta inmune a través de la manipulación de los neutrófilos o de la inhibición de la respuesta de macrófagos.

Por otro lado, se ha detectado a través del fluido del lavado broncoalveolar (LBA) de pacientes con COVID-19 que existe disbiosis de origen oral y del tracto respiratorio superior. ${ }^{65}$ Algunas de las familias detectadas en el fluido LBA son Capnocytophaga y Veillonella, ambas de origen predominantemente oral. ${ }^{2}$ De manera interesante, se ha observado que la abundancia de Fusobacterium periodonticum, bacteria importante dentro de la $\mathrm{EP}$, disminuye tres días después de la infección. ${ }^{66}$ En estos casos, también se ha detectado la expresión de ACE2 en glándula salival, que aumenta conforme la edad. ${ }^{39}$

La ventilación mecánica, a la que pacientes con hipoxia tienen que ser sometidos, está altamente asociada con neumonía. Durante el proceso de intubación, bacterias de la cavidad oral pueden migrar hacia los pulmones. ${ }^{12}$ En este aspecto, un análisis de interacciones señala que las proteínas del género Prevotella pueden promover la infección viral. Este estudio también muestra que proteínas de Prevotella, mas no las del SARS-CoV-2, mantienen interacciones con NF- $\mathrm{B}$, importante promotor de la inflamación y de la severidad clínica durante la COVID-19 (Figura 1). ${ }^{67}$

1. Wang D, Hu B, Hu C, Zhu F, Liu X, Zhang J et al. Clinical characteristics of 138 hospitalized patients with 2019 novel coronavirus-infected pneumonia in Wuhan, China. JAMA [Internet]. 2020 Mar 17;323(11):1061. Available from: https://jamanetwork.com/ journals/jama/fullarticle/2761044

2. Johns Hopkins University. Coronavirus Resource Center [Internet]. 2020 [cited 2020 Dec 12]. Available from: https://coronavirus.jhu.edu/map.html 
3. Dirección General de Epidemiología. Covid-19 México [Internet]. 2020 [cited 2020 Dec 12]. Disponible en: https://datos.covid-19. conacyt.mx/

4. Kinane DF, Stathopoulou PG, Papapanou PN. Periodontal diseases. Nat Rev Dis Prim [Internet]. 2017; 3: 1-14. Available from: http://dx.doi.org/10.1038/nrdp.2017.38

5. Ortiz-García Y, García-Iglesias T, Hernández-Hernández A, Morales V, Cervantes-Cabrera J, Zamora-Pérez A. Factor inhibidor de la migración de macrófagos en la enfermedad periodontal. Rev Mex Periodontol. 2018; 9 (1-2): 14-19.

6. Borges-Yáñez SA, Irigoyen-Camacho ME, Maupomé G. Risk factors and prevalence of periodontitis in community-dwelling elders in Mexico. J Clin Periodontol. 2006; 33 (3): 184-194.

7. Rojo BNR, Flores EA, Arcos CM. Prevalencia, severidad y extensión de periodontitis crónica. Rev Odont Mex. 2011; 15 (1): 31-39.

8. Orozco JR, Peralta LH, Palma MG, Pérez RE, Arróniz PS, Llamosas HE. Prevalencia de gingivitis en adolescentes en el municipio de Tlalnepantla. Rev ADM. 2002; 59 (1): 16-21.

9. Irigoyen ME, Velázquez C, Zepeda MA, Mejía A. Caries dental y enfermedad periodontal en un grupo de personas de 60 o más años de edad de la Ciudad de México. Rev ADM. 1999; 56 (2): 64-69.

10. Medina-Solís CE, Ávila-Burgos L, Hidalgo D. Políticas de salud bucal en México: Disminuir las principales enfermedades. Una descripción. Rev Biomed. 2006; 17 (4): 269-286.

11. Dirección General de Epidemiología. Resultados del Sistema de Vigilancia Epidemiológica de Patologías Bucales SIVEPAB 2019. 2019.

12. Bao L, Zhang C, Dong J, Zhao L, Li Y, Sun J. Oral Microbiome and SARS-CoV-2: Beware of Lung Co-infection. Front Microbiol. 2020; 11: 1-13.

13. Hoffmann M, Kleine-Weber $H$, Schroeder $S$, Krüger $N$, Herrler $\mathrm{T}$, Erichsen $\mathrm{S}$ et al. SARS-CoV-2 cell entry depends on ACE2 and TMPRSS2 and is blocked by a clinically proven protease inhibitor. Cell. 2020; 181 (2): 271-280.e8.

14. Hoffmann M, Kleine-Weber H, Pohlmann S. A multibasic cleavage site in the spike protein of SARS-CoV-2 is essential for infection of human lung cells. Mol Cell. 2020; 78 (4): 779-784.e5.

15. Bello-Chavolla OY, Bahena-López JP, Antonio-Villa NE, VargasVázquez A, González-Díaz A, Márquez-Salinas A et al. Predicting mortality due to SARS-CoV-2: a mechanistic score relating obesity and diabetes to COVID-19 outcomes in Mexico. J Clin Endocrinol Metab. 2020; 105 (8): 2752-2761.

16. Hernández-Galdamez DR, González-Block MÁ, Romo-Dueñas DK, Lima-Morales R, Hernández-Vicente IA, Lumbreras-Guzmán $M$ et al. Increased risk of hospitalization and death in patients with covid-19 and pre-existing noncommunicable diseases and modifiable risk factors in Mexico. Arch Med Res. 2020; 51 (7): 683-639.

17. Mancini L, Quinzi V, Mummolo S, Marzo G, Marchetti E. Angiotensin-converting enzyme 2 as a possible correlation between $\mathrm{CO}$ VID-19 and periodontal disease. Appl Sci. 2020; 10 (18): 6224

18. Kara C, Celen K, Dede FO, Gokmenoglu C, Kara NB. Is periodontal disease a risk factor for developing severe Covid-19 infection? The potential role of Galectin-3. Exp Biol Med. 2020; 245 (16): 1425-1427.

19. Madapusi Balaji T, Varadarajan S, Rao USV, Raj AT, Patil S, Arakeri $G$ et al. Oral cancer and periodontal disease increase the risk of COVID 19? A mechanism mediated through furin and cathepsin overexpression. Med Hypotheses [Internet]. 2020; 144: 109936. Available from: https://linkinghub.elsevier.com/retrieve/pii/ S0306987720311476

20. Polak D, Sanui T, Nishimura F, Shapira L. Diabetes as a risk factor for periodontal disease-plausible mechanisms. Periodontol 2000. 2020; 83 (1): 46-58.

21. Martinez-Herrera M, Silvestre-Rangil J, Silvestre FJ. Association between obesity and periodontal disease. A systematic review of epidemiological studies and controlled clinical trials. Med Oral Patol Oral Cir Bucal. 2017; 22 (6): e708-715.

22. Chaffee BW, Weston SJ. Association between chronic periodontal disease and obesity: a systematic review and meta-analysis. J Periodontol. 2010; 81 (12): 1708-1724.

23. Genco RJ, Grossi SG, Ho A, Nishimura F, Murayama Y. A proposed model linking inflammation to obesity, diabetes, and periodontal infections. J Periodontol. 2005; 76 (11-s): 2075-2084. Available from: http://doi.wiley.com/10.1902/jop.2005.76.11-S.2075

24. Wahid A, Chaudhry S, Ehsan A, Butt S, Khan AA. Bidirectional relationship between chronic kidney disease \& periodontal disease. Pakistan J Med Sci. 2012; 29 (1): 211-215.

25. Ioannidou E, Hall Y, Swede H, Himmelfarb J. Periodontitis associated with chronic kidney disease among Mexican Americans. J Public Health Dent [Internet]. 2013; 73 (2): 112-119. Available from: http://doi.wiley.com/10.1111/j.1752-7325.2012.00350.x

26. De la Rosa E, Irigoyen M, Aranda S, Cruz S, Mondragón A. Enfermedad periodontal en pacientes diabéticos con y sin insuficiencia renal crónica. Rev Med Inst Mex Seguro Soc [Internet]. 2007; 45 (5): 437-446. Disponible en: https://pdfs.semanticscholar.org/3b 6e/868094a63e4d23a637b1030769b85a39fa2a.pdf

27. Hobbins S, Chapple I, Sapey E, Stockley R. Is periodontitis a comorbidity of COPD or can associations be explained by shared risk factors/behaviors? Int J Chron Obstruct Pulmon Dis [Internet] 2017; 12: 1339-1349. Available from: https://www.dovepress. com/is-periodontitis-a-comorbidity-of-copd-or-can-associationsbe-explaine-peer-reviewed-article-COPD

28. Scannapieco FA, Bush RB, Paju S. Associations between periodontal disease and risk for nosocomial bacterial pneumonia and chronic obstructive pulmonary disease. A systematic review. Ann Periodontol. 2003; 8 (1): 54-69. Available from: http://doi.wiley. com/10.1902/annals.2003.8.1.54

29. Hajishengallis E, Hajishengallis G. Neutrophil Homeostasis and Periodontal Health in Children and Adults. J Dent Res [Internet]. 2014; 93 (3): 231-237. Available from: http://journals.sagepub. com/doi/10.1177/0022034513507956

30. Scott DA, Krauss JL. Neutrophils in periodontal inflammation. In: Periodontal disease [Internet]. 2011. 56-83. Available from https://www.karger.com/Article/FullText/329672

31. Usher AK, Stockley RA. The link between chronic periodontitis and COPD: a common role for the neutrophil? BMC Med [Internet]. 2013; 11 (1): 241. Available from: http://bmcmedicine. biomedcentral.com/articles/10.1186/1741-7015-11-241

32. Roberts HM, Ling MR, Insall R, Kalna G, Spengler J, Grant MM et al. Impaired neutrophil directional chemotactic accuracy in chronic periodontitis patients. J Clin Periodontol [Internet]. 2015; 42 (1): 1-11. Available from: http://doi.wiley.com/10.1111/jcpe.12326

33. World Health Organization. Classification of diabetes mellitus. Geneva; 2019.

34. Basto-Abreu A, Barrientos-Gutiérrez T, Rojas-Martínez R, AguilarSalinas CA, López-Olmedo N, De la Cruz-Góngora V et al. Prevalencia de diabetes y descontrol glucémico en Mexico: resultados de la Ensanut 2016. Salud Publica Mex. 2020; 62 (1): 50-59.

35. Preshaw PM, Alba AL, Herrera D, Jepsen S, Konstantinidis A, Makrilakis $\mathrm{K}$ et al. Periodontitis and diabetes: a two-way relationship. Diabetologia. 2012; 55 (1): 21-31.

36. Parohan M, Yaghoubi S, Seraji A, Javanbakht MH, Sarraf P, Djalali M. Risk factors for mortality in patients with Coronavirus disease 2019 (COVID-19) infection: a systematic review and meta-analysis of observational studies. Aging Male. 2020; 23 (5): 1416-1424.

37. Guo W, Li M, Dong Y, Zhou H, Zhang Z, Tian C et al. Diabetes is a risk factor for the progression and prognosis of COVID-19. Diabetes Metab Res Rev. 2020; 36 (7): 1-9.

38. Mantovani A, Byrne CD, Zheng MH, Targher G. Diabetes as a risk factor for greater COVID-19 severity and in-hospital death: 
A meta-analysis of observational studies. Nutr Metab Cardiovasc Dis [Internet]. 2020; 30 (8): 1236-1248. Available from: https:// doi.org/10.1016/j.numecd.2020.05.014

39. Li Y, Xu Q, Ma L, Wu D, Gao J, Chen G et al. Systematic profiling of ACE2 expression in diverse physiological and pathological conditions for COVID-19/SARS-CoV-2. J Cell Mol Med. 2020; 24 (16): 9478-9482.

40. Patel VB, Parajuli N, Oudit GY. Role of angiotensin-converting enzyme 2 (ACE2) in diabetic cardiovascular complications. Clin Sci. 2014; 126 (7): 471-482.

41. Fernandez C, Rysa J, Almgren P, Nilsson J, Engström G, Orho-Melander $\mathrm{M}$ et al. Plasma levels of the proprotein convertase furin and incidence of diabetes and mortality. J Intern Med. 2018; 284 (4): 377-387

42. Oliveira SHP, Brito VGB, Frasnelli SCT, Ribeiro B da S, Ferreira $\mathrm{MN}$, Queiroz DP et al. Aliskiren attenuates the inflammatory response and wound healing process in diabetic mice with periodontal disease. Front Pharmacol. 2019; 10: 1-16.

43. World Health Organization. Diet, nutrition and the prevention of chronic diseases. World Health Organization technical report series. Vol. 916, Switzerland; 2003.

44. Huttunen R, Syrjanen J. Obesity and the risk and outcome of infection. Int J Obes. 2013; 37 (3): 333-340.

45. Simonnet A, Chetboun M, Poissy J, Raverdy V, Noulette J, Duhamel A et al. High prevalence of obesity in severe acute respiratory syndrome coronavirus-2 (SARS-CoV-2) requiring invasive mechanical ventilation. Obesity. 2020; 28 (7): 1195-1199.

46. Sheridan PA, Paich HA, Handy J, Karlsson EA, Hudgens MG, Sammon $A B$ et al. Obesity is associated with impaired immune response to influenza vaccination in humans. Int J Obes [Internet]. 2012; 36 (8): 1072-1077. Available from: http://dx.doi. org/10.1038/ijo.2011.208

47. Jia X, Yin C, Lu S, Chen Y, Liu Q, Bai J et al. Two things about COVID-19 might need attention. Preprints. 2020; 2:

48. Araújo MVF, Hong BY, Fava PL, Khan S, Burleson JA, Fares G et al. End stage renal disease as a modifier of the periodontal microbiome. BMC Nephrol. 2015; 16 (1): 1-7.

49. Wang B, Li R, Lu Z, Huang Y. Does comorbidity increase the risk of patients with COVID-19. Aging (Albany NY). 2020; 12 (7): 6049-6057.

50. Henry BM, Lippi G. Chronic kidney disease is associated with severe coronavirus disease 2019 (COVID-19) infection. Int Urol Nephrol [Internet]. 2020; 52 (6): 1193-1194. Available from: https://doi.org/10.1007/s11255-020-02451-9

51. From the Global Strategy for the Diagnosis, Management and Prevention of COPD GI for COLD (Gold) 2017. AFHO. Guías para el diagnóstico y tratamiento de la enfermedad pulmonar obstructiva crónica. Neumol Cir Torax. 2012; 71 (1): 1-89.

52. Scannapieco FA, Ho AW. Potential associations between chronic respiratory disease and periodontal disease: analysis of national health and nutrition examination survey III. J Periodontol [Internet]. 2001; 72 (1): 50-56. Available from: http://doi.wiley. com/10.1902/jop.2001.72.1.50

53. Scannapieco FA. Individuals with chronic obstructive pulmonary disease (COPD) may be more likely to have more severe periodontal disease than individuals without COPD. J Evid Based Dent Pract [Internet]. 2014; 14 (2): 79-81. Available from: https://linkinghub.elsevier.com/retrieve/pii/S1532338214000761

54. Hoenderdos K, Condliffe A. The neutrophil in chronic obstructive pulmonary disease. too little, too late or too much, too soon? Am J Respir Cell Mol Biol [Internet]. 2013; 48 (5): 531-539. Available from: http://www.atsjournals.org/doi/abs/10.1165/rcmb.20120492TR

55. Burnett D, Hill S, Chamba A, Stockley R. Neutrophils from subjects with chronic obstructive lung disease show enhanced chemotaxis and extracellular proteolysis. Lancet [Internet]. 1987;
330 (8567): 1043-1046. Available from: https://linkinghub.elsevier.com/retrieve/pii/S0140673687914760

56. Zhao Q, Meng M, Kumar R, Wu Y, Huang J, Lian $N$ et al. The impact of COPD and smoking history on the severity of COVID-19: A systemic review and meta-analysis. J Med Virol [Internet]. 2020; 92 (10): 1915-1921. Available from: https://onlinelibrary. wiley.com/doi/10.1002/jmv.25889

57. Alqahtani JS, Oyelade T, Aldhahir AM, Alghamdi SM, Almehmadi $M$, Alqahtani AS et al. Prevalence, severity and mortality associated with COPD and smoking in patients with COVID-19: a rapid systematic review and meta-analysis. Bhatt GC, editor. PLoS One [Internet]. 2020; 15 (5): e0233147. Available from: https:// dx.plos.org/10.1371/journal.pone.0233147

58. Leung JM, Yang CX, Tam A, Shaipanich T, Hackett TL, Singhera GK et al. ACE-2 expression in the small airway epithelia of smokers and COPD patients: implications for COVID-19. Eur Respir J [Internet]. 2020; 55 (5): 2000688. Available from: http:// erj.ersjournals.com/lookup/doi/10.1183/13993003.00688-2020

59. Maremanda KP, Sundar IK, Li D, Rahman I. Age-dependent assessment of genes involved in cellular senescence, telomere, and mitochondrial pathways in human lung tissue of smokers, COPD, and IPF: associations with SARS-CoV-2 COVID-19 ACE2TMPRSS2-Furin-DPP4 Axis. Front Pharmacol. 2020; 11: 1-16.

60. Cekici A, Kantarci A, Hasturk H, Van Dyke TE. Inflammatory and immune pathways in the pathogenesis of periodontal disease. Periodontol 2000. 2014; 64 (1): 57-80.

61. Hajishengallis G, Lamont RJ. Beyond the red complex and into more complexity: The polymicrobial synergy and dysbiosis (PSD) model of periodontal disease etiology. Mol Oral Microbiol. 2012; 27 (6): 409-419.

62. Lamont RJ, Koo H, Hajishengallis G. The oral microbiota: dynamic communities and host interactions [Internet]. Vol. 16, Nature Reviews Microbiology. Springer US; 2018, 745-759. Available from: http://dx.doi.org/10.1038/s41579-018-0089-x

63. Ai D, Huang R, Wen J, Li C, Zhu J, Xia LC. Integrated metagenomic data analysis demonstrates that a loss of diversity in oral microbiota is associated with periodontitis. BMC Genomics [Internet]. 2017; 18 (Suppl 1): 1-15. Available from: http://dx.doi. org/10.1186/s12864-016-3254-5

64. Duran-Pinedo AE, Chen T, Teles R, Starr JR, Wang X, Krishnan $\mathrm{K}$ et al. Community-wide transcriptome of the oral microbiome in subjects with and without periodontitis. ISME J. 2014; 8 (8): 1659-1672.

65. Shen Z, Xiao Y, Kang L, Ma W, Shi L, Zhang L et al. Genomic diversity of severe acute respiratory syndrome-coronavirus 2 in patients with coronavirus disease 2019. Clin Infect Dis [Internet]. 2020; 71 (15): 713-720. Available from: https://academic.oup. com/cid/article/71/15/713/5780800

66. Moore SC, Penrice-Randal R, Alruwaili M, Dong X, Pullan ST, Carter DP et al. Amplicon based MinION sequencing of SARSCoV-2 and metagenomic characterisation of nasopharyngeal swabs from patients with COVID-19. medRxiv [Internet]. 2020 2020.03.05.20032011. Available from: http://medrxiv.org/content/early/2020/03/08/2020.03.05.20032011.abstract

67. Khan AA, Khan Z. COVID-2019-associated overexpressed Prevotella proteins mediated host-pathogen interactions and their role in coronavirus outbreak. Bioinformatics. 2020; 36 (13): 4065-4069.

Conflicto de intereses: Los autores declaran no tener algún conflicto de intereses.

\section{Correspondencia: \\ Tonatiuh Abimael Baltazar-Díaz}

E-mail: tonatiuhabd@gmail.com 